\title{
Sea-wave Dynamic Loading of Sailing Yacht's Retractable Keel
}

\author{
Pino Koc* \\ University of Ljubljana, Faculty of Mathematics and Physics, Slovenia
}

A highly specialized analysis of functionality of a retractable keel lifting mechanism is presented in the paper. Because of its special design a retractable keel is vulnerable to rough sea conditions. To alleviate possible malfunctions or even damage to the keel, an analysis of lifting the keel during rough sea (WMO sea state 6) is performed.

Reliable estimation of load acting on a keel is crucial for the subsequent functional or structural analysis of the keel. Aiming to obtain these loads, a sea wave analysis is performed first, where a wave train is determined by respecting statistical data regarding the required sea condition. Two wave cases are considered: head seas (waves coming from the front) and beam seas (lateral waves). A deterministic dynamic analysis on the wave-hull-keel system is then performed. The response of this system is obtained in the form of a time dependent acceleration of the centre of gravity of the vessel and in a time dependent lifting force, i.e., the force which is required for lifting the moveable part of the keel during rough sea. Finally, the required pulling force of the lifting mechanism is determined.

Keywords: sailing yacht, retractable keel, wind wave, computer simulation

\section{INTRODUCTION}

One of the tasks of a monohull sailing yacht's keel is to compensate for yacht heeling due to the wind force. The deeper and heavier the keel is, the smaller the heeling angle or, viewed from another angle, for the same heeling angle more sails can be used. Too deep of a keel prohibits access to shallow waters and harbours. A retractable keel is the solution to this problem and still provides a good sailing ability. From a mechanical point of view, several kinematic mechanisms are possible to retract a keel. One special type of a retractable keel, used for cruising sailing yachts has been developed in recent years and is manufactured now in some shipyards worldwide [1]. It is a so-called telescopic lifting keel that is located completely outside (below) the hull. The advantage of this type of keel is that it does not influence the interior of the hull nor does it limit the cabin arrangements.

The working principle of a telescopic keel is shown in Fig. 1. The keel consists of a fixed part bolted to the hull structure, a moveable part which slides along guides embedded in a fixed part, and a hydraulic cylinder for lifting and lowering the moving part. The lifting height is relatively small as compared to more common lifting keels which intrude into the hull, at the most $1 \mathrm{~m}$. Since it is desirable to have a heavy keel, it is fabricated of cast iron and thick steel weldments. Although made of heavy sections which give the illusion of indestructibleness, a telescopic keel is in fact a mechanism exposed to a rough seawater environment and seafaring conditions, and is thus prone to various types of break-downs and damage.

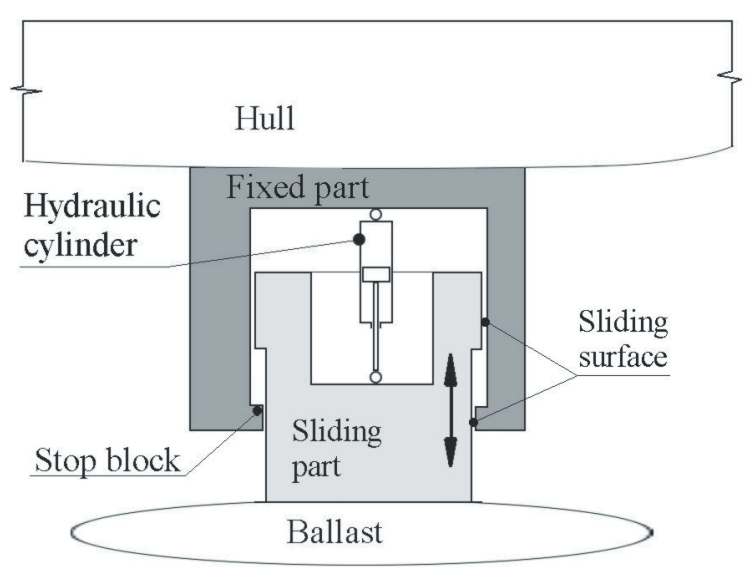

Fig. 1. Working principle of the telescopic lifting keel

An important step in an attempt to avoid any malfunction of the telescopic keel is a reliable functional and structural analysis.

\section{PROBLEM DEFINITION}

Basic mechanical design and a structural qualification of an ordinary, i.e., fixed keel is a well-known task, based on simple mechanical principles [2] and verified through many successful designs. When designing more complicated types of keels, lifting or canting, for example, advanced designing methods, based on finite element (FE) analysis are inevitable [3]. Also, fixed or retractable keel hydraulic design is a highly demanding task and only in the last few decades has it been thoroughly comprehended through the aid of modern computational techniques [4]. 
In the structural analysis of the retractable keel, peculiarities such as sea-wave dynamic loading, extreme events (capsizing, grounding and collision), geometrical (intermittent contact between moveable and fixed parts of keel) and material (plastic deformation) nonlinearities and force variability in a hydraulic cylinder need to be considered. As such, a presentation of the whole structural analysis is too extensive; therefore, only a part of it will be presented in the paper.

The response of the hydraulic cylinder during rough water loading conditions is of a special interest for the designer. It is required that the cylinder is able to retract the moving parts of the keel even in a foul weather, i.e., in our case at the sea state scale 6, according to the World Meteorological Organization (WMO). At that sea state, the Significant Wave Height (SWH or traditionally $H_{1 / 3}$ ) is between 4 to $6 \mathrm{~m}$. The rest of the input data, used in this work are:

- moveable mass (moveable part of the keel plus ballast): app. 5,500 kg;

- cylinder stroke, i.e., lifting height: $500 \mathrm{~mm}$;

- duration of lifting: $60 \mathrm{~s}$;

- yacht's maximal speed during lifting: $1.54 \mathrm{~m} / \mathrm{s}$ (3 knots); and

- wave direction: Case 1: from the front (head seas) and Case 2: from the side (beam seas).

The goal of analysis is to predict the minimal cylinder force which is needed to accomplish the above defined task of retracting the keel on a rough sea.

\section{SEA-WAVE ANALYSIS}

Note: Sea waves are primarily a consequence of the wind blowing along the sea's surface; therefore, they are also sometimes called wind waves. In the sequel of this paper, the term sea wave will be used.

Sea waves shape and floating body shape, mass and mass inertia influence the dynamic response of that body. A common approach is to evaluate wave characteristics through statistical means, using predefined wave spectra. An exact shape of the wave train cannot be retrieved from these analyses, which is not needed if a special kind of probabilistic analysis developed for naval engineering, so-called seakeeping analysis [5], is performed.

In a structural analysis of the retractable keel, based on a deterministic approach of classical mechanics, a shape of the wave train, i.e., time and space dependence of the wave height needs to be known at the beginning of the analysis. Since we do not posses records of wave measurements, a pure mathematical approach [6] is used in our work. It is based on the fact that by a superposition of many sinusoidal waves, a quasi irregular wave pattern, similar to that of the natural wave train, can be obtained.

At the arbitrary point $(x, y)$ on the sea surface and at the time $t$, the instantaneous height of the surface $h$, measured from the still water level is:

$$
\begin{aligned}
& h(x, y, t)= \\
& =\sum_{i=1}^{n} h_{A i} \cos \left(\frac{2 \pi x}{\lambda_{i}} \cos \alpha_{i}+\frac{2 \pi y}{\lambda_{i}} \sin \alpha_{i}-\omega_{i} t+\varphi_{i}\right),
\end{aligned}
$$

where $n$ is the number of superimposed sine waves, $h_{A i}, \lambda_{i}$ and $\alpha_{i}$ represent the amplitude (half of the wave height $H$ ), wavelength and wave propagation direction (Fig. 2) of $i^{\text {th }}$ sine wave respectively. $\varphi_{i}$ is the phase shift and $\omega_{i}$ is the rotational frequency of $i^{\text {th }}$ wave

$$
\omega_{i}=\sqrt{\frac{2 \pi g}{\lambda_{i}}}
$$

with $g$ as a gravitational acceleration constant.

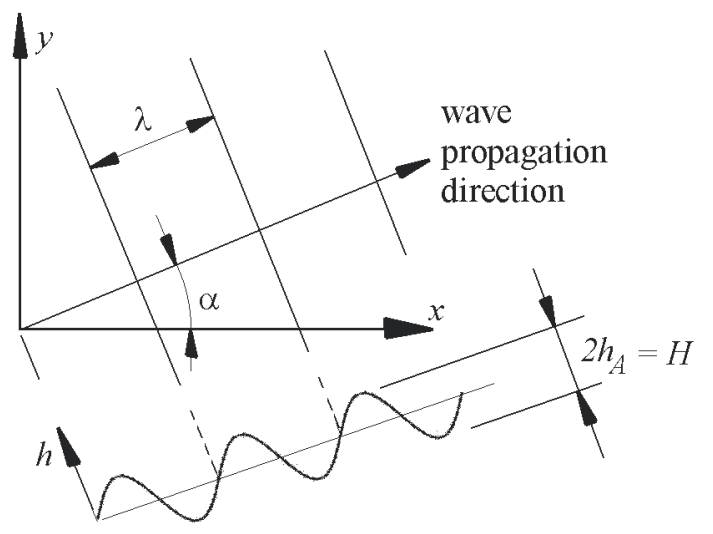

Fig. 2. Sinusoidal wave characteristics

By estimating the natural frequency of a yacht's rolling and pitching (angular oscillation about longitudinal and lateral axis, respectively) and in concordance with [7], a combination of waves which leads to a strong motion of the yacht was found. Three sinusoidal waves $(n=3)$ are assembled with their individual characteristics shown in Table 1.

A result of the superposition is the time dependent undulate surface of the sea. In Figs. 7 and 8 this surface is shown at several arbitrary chosen time instances. To check some statistical characteristics of artificial formed wave surface, two plots are 


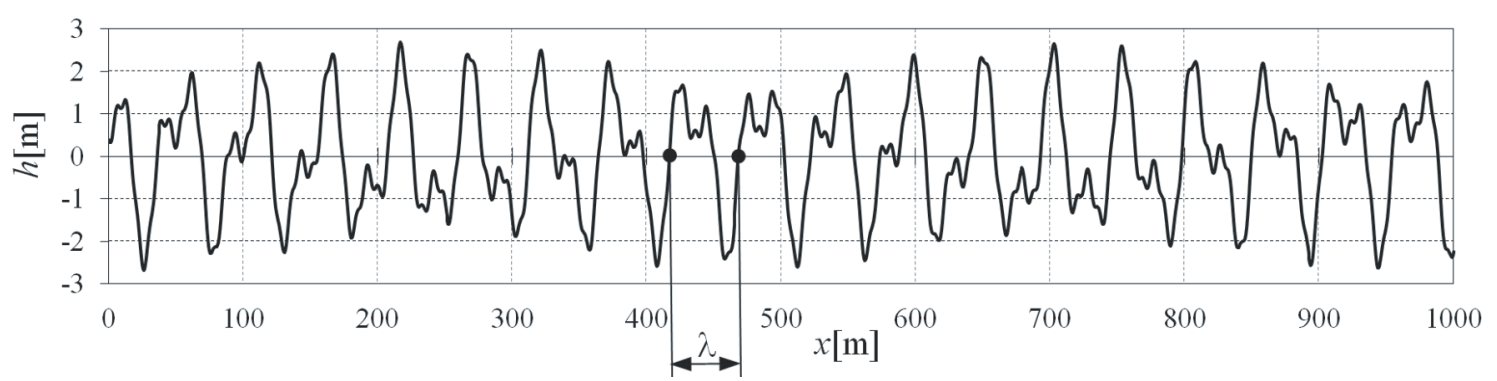

Fig. 3. Height of the artificial wave along a line $y=0 \mathrm{~m}$ and at $t=0 \mathrm{~s}$

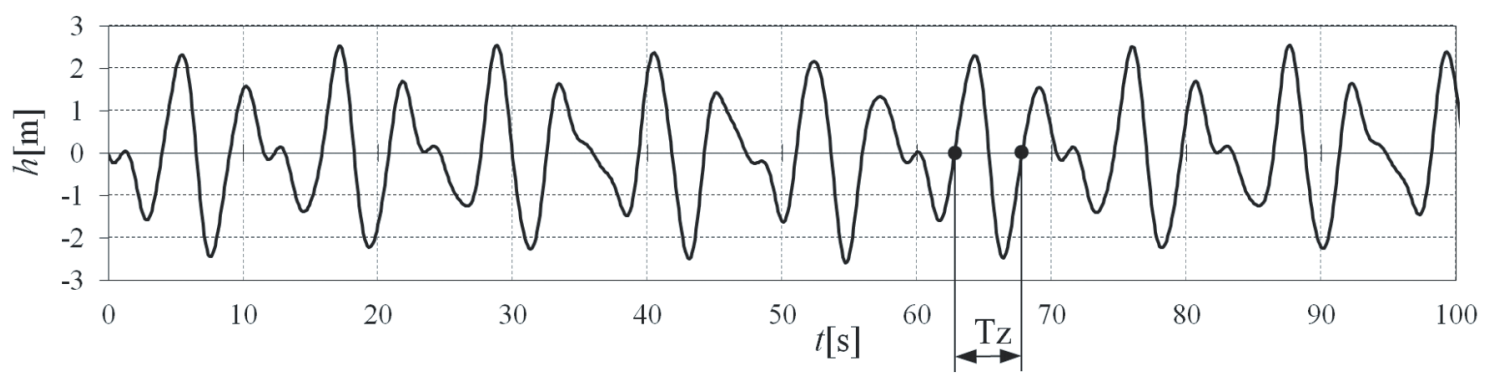

Fig. 4. Time variation of height of the artificial wave at $x=100 \mathrm{~m}$ and $y=0 \mathrm{~m}$

given, where spatial, i.e., along a line (Fig. 3) and time (Fig. 4) dependence of artificial wave height $h$ is displayed. From Fig. 3, a kind of periodicity can be observed. But shapes of individual waves are not repeating within $1,000 \mathrm{~m}$ of wave path. An average wavelength $\lambda$ of the prominent waves is about $54 \mathrm{~m}$. In Fig. 4, a much more regular wave pattern is seen. The period of repeating the pattern is about $60 \mathrm{~s}$. A so-called zero crossing period $T z$ is shown in Fig. 4. An average $T z=5.8 \mathrm{~s}$ is measured from the graph. The wave spectra derived from Fig. 4 would not be a smooth curve as in case of natural sea waves [5], but it would consists of three distinctive peaks of equal heights, the first at about 0.4 , the second at 3 and the third at $4.8 \mathrm{~m}$. Therefore, the significant wave height $H_{1 / 3}$, defined as the average height of the waves which comprise top $33 \%$ of all waves, equals to $H_{1 / 3}=4.8 \mathrm{~m}$.

Table 1. Sinusoidal wave data

\begin{tabular}{ccccc}
\hline$i$ & $\lambda[\mathrm{m}]$ & $h_{A}[\mathrm{~m}]$ & $\alpha\left[^{\circ}\right]$ & $\varphi\left[^{\circ}\right]$ \\
\hline 1 & 54 & 1.5 & 0 & 0 \\
\hline 2 & 24 & 1.0 & -20 & 175 \\
\hline 3 & 8 & 0.2 & 10 & 130 \\
\hline
\end{tabular}

Characteristic wave data obtained above are compared with data at WMO scale 6, given in [8]: $H_{1 / 3}=4.6 \div 5.9 \mathrm{~m}, \lambda=46.5 \div 55 \mathrm{~m}$, and $\mathrm{Tz}=8.3 \div 9.0 \mathrm{~s}$. We can conclude that artificial wave data $H_{1 / 3}$ and $\lambda$ match data from [8], while $T z$, obtained from Fig. 4, is shorter. This means that a vessel with a length smaller than average wavelength $\lambda$ will encounter crests of artificial waves at an increased rate, producing more violent movement of the vessel, which is conservative from the structural analysis point of view.

\section{NUMERICAL MODEL}

A finite element-based computer code ABAQUS [9] is used for analysis of the yacht's dynamic response. A mechanical scheme of the yacht's model is given in Fig. 5.

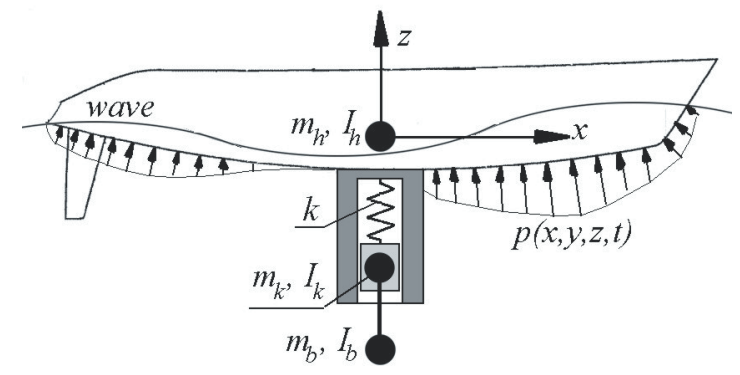

Fig. 5. Mechanical scheme of wave-hull-keel system

Since the main goal of the analysis is to determine the hydraulic cylinder lifting capability, the hull, the fixed and moveable parts of the telescopic keel and the ballast are considered as rigid bodies. The total mass $m_{h}$ of the hull with all appendages except for the moveable parts of the keel is $20,700 \mathrm{~kg}$ and is applied in the centre of gravity (CG) of the hull. Masses of the 
moveable part of the keel $m_{k}$ and the ballast $m_{b}$ are $1,100 \mathrm{~kg}$ and 4,400 $\mathrm{kg}$, respectively, and are applied in their respective centres of gravity as well. To adequately capture rotational oscillations of the yacht about three spatial axes (roll, pitch and yaw) mass moments of inertia are calculated for the hull, both parts of the keel and the ballast $\left(I_{h}, I_{k}\right.$ and $I_{b}$ in Fig. 5).

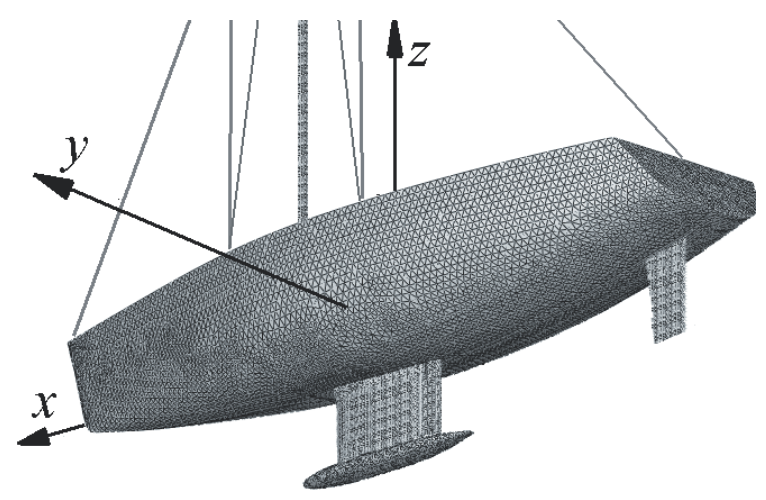

Fig. 6. FE mesh of the sailing yacht

Because the shape of the hull influences intensity and direction of static and dynamic water pressure, it is reproduced in the FE model as exactly as possible (Fig. 6). In the model, the hull is massless and is rigidly connected with mass $m_{h}$. Some general data of the yacht are: hull length $18 \mathrm{~m}$ and draft $2.5 \mathrm{~m}$ (lower position of the lifting keel).

The moveable part of the keel and the ballast are rigidly connected. This combined rigid body is kinematically restrained to translate along the sliding direction only (vertical direction in Fig. 5).

The hydraulic cylinder inside the telescopic keel is modelled with deformable beam elements to allow extension/shortening of the cylinder and is designated as spring $k$ in Fig. 5. Also, the stainless steel guides and reinforced polymer padding which constitute contact surfaces are modelled as deformable bodies due to their importance in the dynamic response of the lifting keel.

Loads acting on the yacht are gravity and wave pressure. Since it is not allowed to be lifting the keel when the yacht is heeled due to the sails, no wind forces on the sails need to be considered in analysis.

Water pressure $p(x, y, z, t)$ on the hull is not stationary when the yacht is on the waves. To model nontypical loadings, ABAQUS offers subroutine DLOAD [9], which is used in our case to model the wave hydrodynamic phenomena:

- The wave surface is modelled as described in Section 2, Eq. (1).
- Water pressure in the wave depends on the orbital velocity of the water particle and is smaller in the wave crest than in trough [10]. A linear variation of the pressure with the depth (vertical distance from the wave surface) is adopted in this research. This means that a higher buoyancy force is modelled on the wave crest, thus promoting pitching and heaving (i.e., translation oscillations in a vertical direction) of the yacht in case of head seas, which is conservative from a structural analysis aspect.

- Slamming of the hull against the water surface is considered one of the worst dynamic loadings (except touching another hard object) [2] and therefore need to be accounted for. The slamming pressure is calculated based on a hull-to-water surface velocity difference.

The water drag of the hull and the keel is neglected due to the small speed during keel lifting, as defined above.

When the vessel is on the waves, damping plays a crucial role attaining a vessel's stability and seaworthiness [10]. In our work, damping is introduced in the model through a so-called artificial damping. Defining a correct amount of damping in such cases is not a trivial task; therefore, we performed a sensitivity analysis of the yacht oscillating on still water. Three of the most important oscillating modes were examined: rolling, pitching and heaving. For each mode, critical damping was found. Damping used in a model is taken as a fraction of the critical damping and takes into account an estimated frequency of natural oscillation.

\section{RESULTS OF NUMERICAL ANALYSIS}

\subsection{Computer Simulation of Yacht's Behaviour on the Waves}

Two directions of wave incidence are considered in the work: the head seas and the beam seas. For both cases, a dynamic analysis of a yacht's response on wave excitation was performed. In Figs. 7 and 8, an illustration of a yacht's response is shown at three arbitrary chosen instances for the head seas case (Fig. 7) and for the beam seas (Fig. 8) simulation.

Since the aim of these particular simulations is to determine the most unfavourable time sequence of yacht's accelerations, no lifting of the keel needs to be simulated. Because of that, the moveable part of the keel can be tied on the fixed part. This means that contact calculations between a keel's parts are deactivated, which in turn considerably speed up the simulation. 
a)

b)
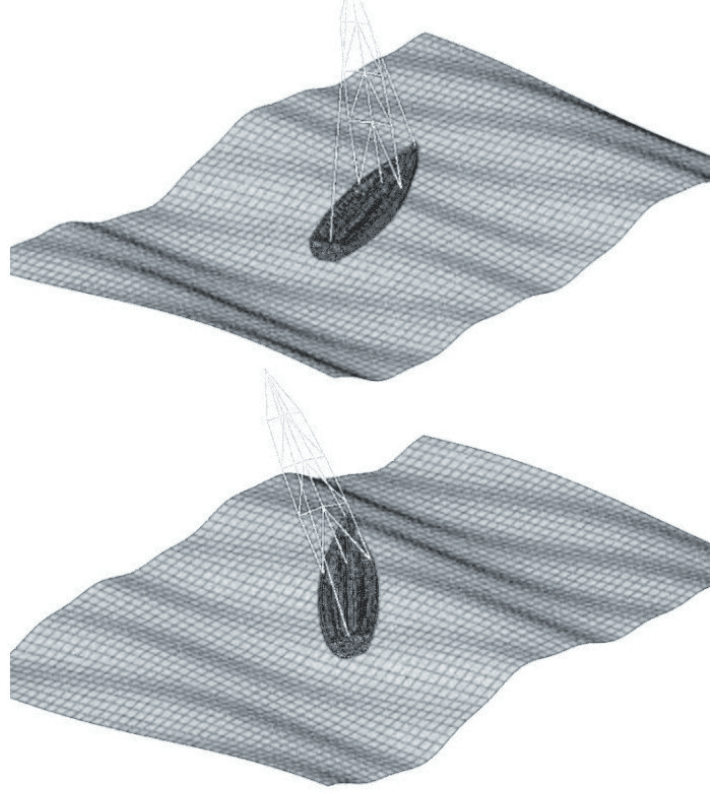

c)

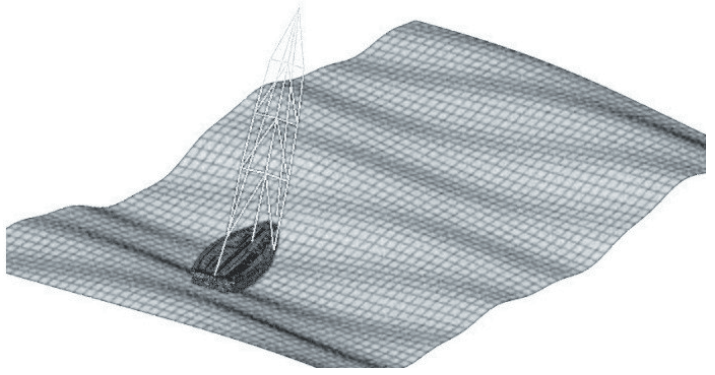

Fig. 7. Yacht on head seas; a) $t=4 \mathrm{~s}$; b) $t=11 \mathrm{~s} ;$ c) $t=38 \mathrm{~s}$

In Figs. 9 and 10, the results of the head seas simulation, i.e., translational and angular accelerations of the centre of gravity of yacht are displayed. It can be seen that some kind of weak periodicity appears every 12 to $14 \mathrm{~s}$. Exemptions are the very first $3 \mathrm{~s}$ of vertical accelerations $(a z)$ and angular acceleration about the $y$ axis, which are altering violently as compared to the rest of the graph. These abrupt changes are attributed to the imperfections of the model. Namely, at the beginning of the simulation, the hull is placed on the crest without checking the static equilibrium of gravity and buoyancy forces. Depending on the resultant force, the hull then falls down on the wave or jumps out of the crest. From diagrams, the prevailing mode of the yacht movement is also seen: heaving and pitching. In case of beam seas another intensive movement is rolling. The same acceleration histories, but for the beam seas case, are given in Figs. 11 and 12.

From the above diagrams, it can be seen that at the same sea state scale 6 , beam seas cause higher a)

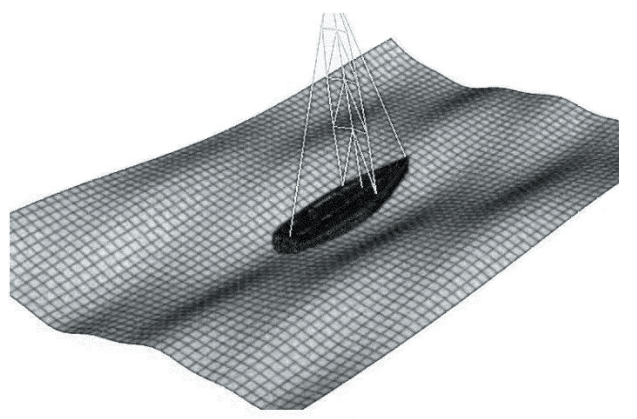

b)

c)
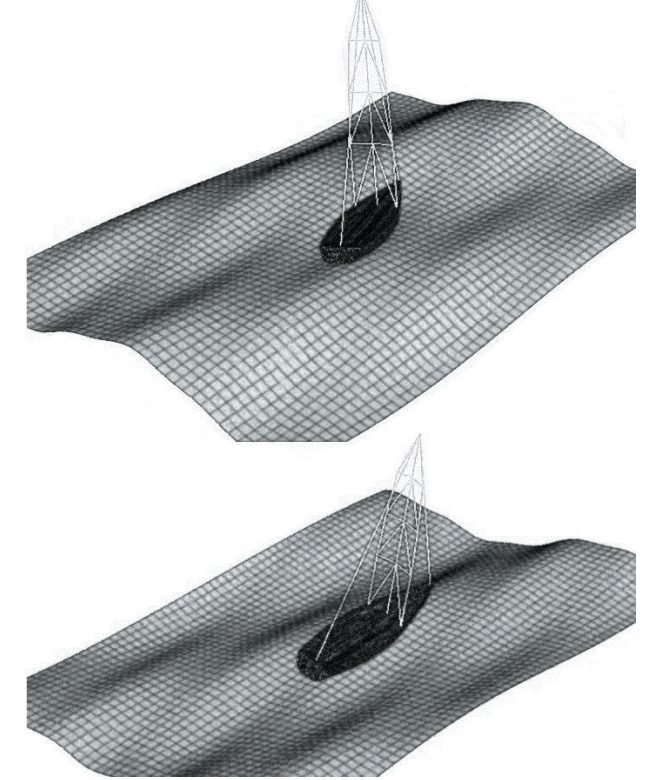

Fig. 8. Yacht on beam seas; a) $t=3 \mathrm{~s} ; b) t=6 \mathrm{~s} ;$ c) $t=12 \mathrm{~s}$

accelerations (translational and angular) of the yacht's centre of gravity than head seas. Therefore, it is appropriate to simulate the lifting of the keel considering the beam sea's response.

\subsection{Simulation of Keel Lifting}

Because the lifting of the keel is simulated, all contacts and friction between the fixed and moveable parts of the keel need to be considered in the simulation. This increases the analysis (CPU) time substantially. Aiming to shorten the analysis time, we decided to simulate the lifting of the keel with a higher lifting speed as prescribed by the design.

The greatest effort that the hydraulic cylinder needs to give is at the beginning of the lifting, since the moveable parts of the keel are then at the greatest distance from the centre of gravity of the yacht. At that position, angular accelerations (about three space axes) of the yacht produce the biggest translational accelerations and, coupled with the shortest distance 
between upper and lower sliding surfaces (Fig. 1), the biggest friction force between the contact surfaces evolve, leading to the biggest lifting force. Therefore, it would be conservative to start the lifting of the keel at the moment when high accelerations (translational and angular) occur. By inspecting the beam seas diagrams in Figs. 11 and 12, the most intensive acceleration time sequence is determined to be between the $9^{\text {th }}$ and $28^{\text {th }}$ second. In the simulation of keel lifting, beam elements representing the hydraulic cylinder are manipulated such that the keel lifting is simulated within the selected time interval.

In Fig. 13, the most important result of lifting simulation, i.e., the axial force in the hydraulic cylinder, is shown. At the beginning of lifting at the $9^{\text {th }}$ second, the piston's rod is compressed as prescribed by the design. Namely, when the keel is in the lower position, it is pressed by the cylinder against the stop block (Fig. 1). This pressure is adequate to close all of the gaps in the polymer-stainless steel contact surface, thus achieving firm contact between the fixed and moveable parts of the keel. Also, eventual lifting of the keel due to wave acceleration is prevented. After the $9^{\text {th }}$ and until the $12^{\text {th }}$ second, forces in the piston gradually change from compressive to tensile. This part of the curve represents a diminishing of pre-

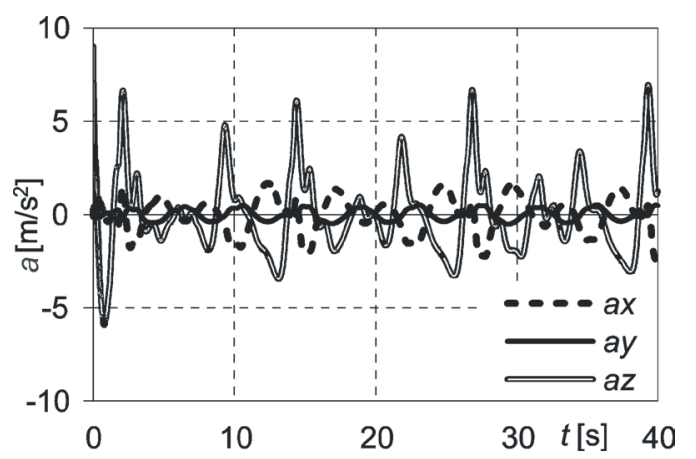

Fig. 9. Translational acceleration of yacht`s $C G$ at head seas

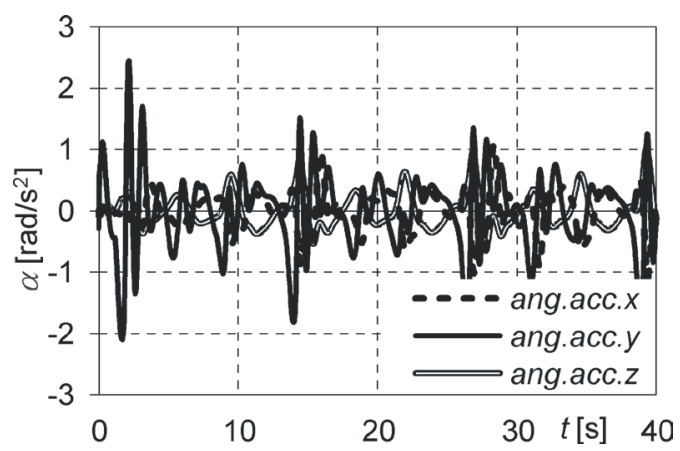

Fig. 10. Angular acceleration of yacht `s $C G$ at head seas compressing of the contact surfaces which finally leads to separation of the contact surfaces. From this point on, the whole weight and other inertial loads, acting in the sliding direction of the moveable keel, are transmitted through the cylinder as a tensile force. After the $12^{\text {th }}$ second, the dynamic response due to wave excitation can be seen. The highest tension force in the cylinder is $160 \mathrm{kN}$. Also, it can be seen that force peaks becoming smaller in the last third of the lifting time and that the force approaches the value of the weight of the moveable part of the keel.

\subsection{Discussion}

Determination of the wave train represents the biggest uncertainty introduced in the analysis. Namely, for the same state of the sea, which is the statistical quantity, an infinite number of deterministic wave trains can be generated. The same problem is encountered by civil engineers when evaluating earthquake effects by deterministic method such as the time integration scheme. Since the earthquake excitation is given as the frequency domain acceleration spectrum, many acceleration histories, which fit into this spectrum, can be generated. Besides repeating analyses with different acceleration histories the usual way is to

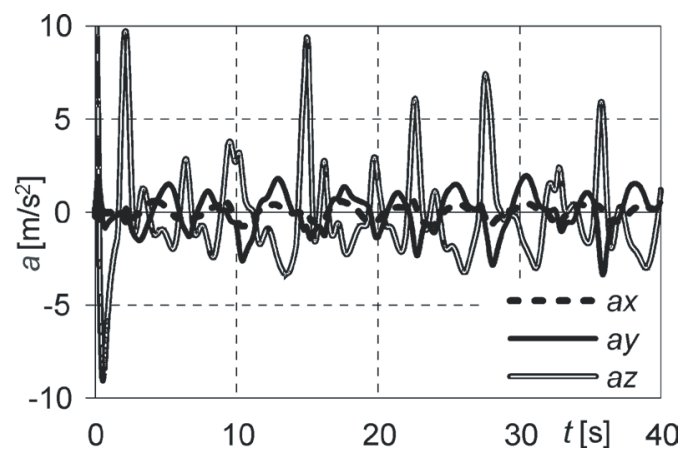

Fig. 11. Translational acceleration of yacht's $C G$ at beam seas

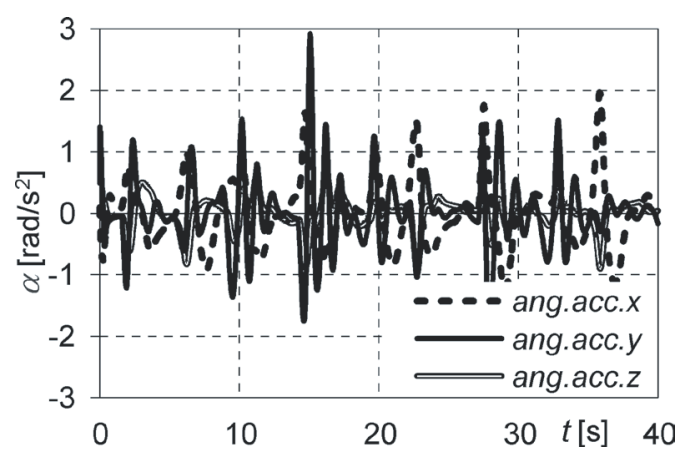

Fig. 12. Angular acceleration of yacht's $C G$ at beam seas 


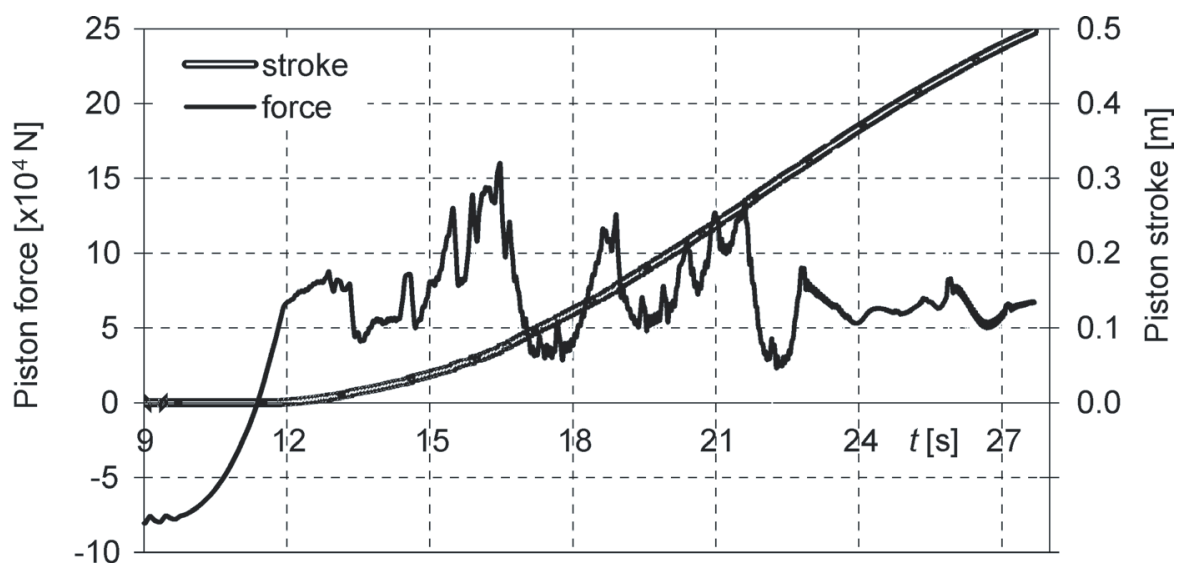

Fig. 13. Piston force and stroke during lifting of the keel

perform only one analysis and then multiply the obtained results by an appropriate scaling factor. While the civil engineering community has some guidelines of how big this factor could be, we do not know for similar guidelines in naval engineering. If simply copying this scaling factor from the civil engineering field then it would be between 1.2 and 1.5. Taking the most conservative value, this means that hydraulic cylinder should be capable of pulling with a force of $1.5 \times 160 \mathrm{kN}=240 \mathrm{kN}$.

\section{CONCLUSIONS}

In the paper, an analysis of hydraulic cylinder capability for lifting the yacht's keel during rough sea conditions is described. The problem is solved without using highly sophisticated computational fluid dynamics (CFD) programs. Because the yacht's velocity relative to the water is small, a substitute method as shown in the paper could be used. The drawback for this is that several assumptions needed to be adopted - usually in the way in which the obtained solution is rather conservative (see Section 3, water pressure loading). Thus, the results are acceptable and serve as a support in the subsequent detailed designing process.

\section{ACKNOWLEDGEMENT}

This work is fully sponsored by the Seaway group. The author wishes to express thanks to the staff of the Seaway group for their technical support during the research.

\section{REFERENCES}

[1] Nautic Expo. Lifting keel sailboats, from: http:// www.nauticexpo.com/boat-manufacturer/lifting-keelsailboat-21398.html, accessed on 2013-09-04.

[2] Larsson, L., Eliasson, R.E. (2000). Principles of Yacht Design, $2^{\text {nd }}$ ed. McGraw-Hill, London, DOI:10.1038/35018068.

[3] Colombo, C., Vergani, L., Modica, N., A. (2007). Numerical and experimental study of a crash absorption keel. Key Engineering Materials, vol. 348-349, p. 953-956, DOI:10.4028/www.scientific.net/KEM.348349.953 .

[4] Stern, F., Yang, J., Wang, Z., Sadat-Hosseini, H., Mousaviraad, M., Bhushan, S. (2012). Computational ship hydrodynamics: nowadays and way forward. Proceedings on 29th Symposium on Naval Hydrodynamics, Gothenburg.

[5] Lloyd, A.R.J.M. (1989) Seakeeping: ship behaviour in rough weather. Ellis Horwood Limited, Chichester, UK.

[6] Ship, behaviour on the waves (1964). Technical Encyclopedia, Volume 2, Miroslav Krleža Lexicographical Institute, Zagreb, p. 228. (in Croatian)

[7] Fridsma, G. (1969). A systematic study of the roughwater performance of planning boats, Report 1275. Davidson laboratory, Stevens Institute of Technology, New Jersey.

[8] Zorović, D., Mohović, R., Mohović, Đ. (2003). Towards determining the length of the wind waves of the Adriatic sea. Journal of Marine Science and Technology, vol. 50, no. 3-4, p. 145-150. (in Croatian)

[9] ABAQUS/Standard, Version 6.7 (2007) User's manual, Simulia, Providence.

[10] Marchay, C.A. (1996). Seaworthiness the Forgotten Factor. Adlard Colles Nautical, London. 